\title{
Primary infection with Chlamydia trachomatis L2c in a porcine model produced urogenital pathology similar as in humans and failed to induce protective immune responses against re-infection
}

\author{
Evelien De Clercq ${ }^{1}$, Matthias Van Gils ${ }^{1}$, Katelijn Schautteet ${ }^{1}$, Bert Devriendt ${ }^{2}$, Koen \\ Chiers $^{3}$, Eric $\mathrm{Cox}^{2}$, Deborah Dean ${ }^{4 *}$ and Daisy Vanrompay ${ }^{1 *}$ \\ ${ }^{1}$ Department of Molecular Biotechnology, Faculty of Bioscience Engineering, Ghent \\ University, Coupure Links 653, B-9000 Ghent, Belgium. ${ }^{2}$ Laboratory of \\ Immunology, Faculty of Veterinary Medicine, Ghent University, Salisburylaan 133, \\ B-9820 Merelbeke, Belgium. ${ }^{3}$ Department of Pathology, Bacteriology and Poultry \\ Diseases, Faculty of Veterinary Medicine, Ghent University, Salisburylaan 133, B- \\ 9820 Merelbeke, Belgium. ${ }^{4}$ Center for Immunobiology and Vaccine Development, \\ Children's Hospital Oakland, Research Institute, Oakland, California, USA. \\ *shared senior authorship
}

We evaluated the pathogenesis, pathology and immune response of a female genital tract infection with Chlamydia trachomatis L2c, the most recently discovered lymphogranuloma venereum strain, using a porcine model of sexually transmitted infection. Pigs were mock infected, infected once or infected and re-infected intravaginally, and samples were obtained for chlamydial culture, gross and microscopic pathology, and humoral and cell-mediated immunity. Intravaginal inoculation of pigs with this bacterium resulted in an infection that was confined to the urogenital tract, where inflammation and pathology were caused that resembled what is seen in human infection. Re-infection resulted in more severe gross pathology than primary infection, and chlamydial colonization of the urogenital tract was similar for primary infected and re-infected pigs. This indicates that primary infection failed to induce protective immune responses against re-infection. Indeed, the proliferative responses of mononuclear cells from blood and lymphoid tissues to C. trachomatis strain L2c were never statistically different among groups, suggesting that $C$. trachomatis-specific lymphocytes were not generated following infection or reinfection. Nevertheless, antichlamydial antibodies were elicited in sera and vaginal secretions after primary infection and re-infection clearly demonstrating a secondary systemic and mucosal antibody response. The porcine model is relevant for evaluating immune and pathogenic responses for emerging and known C. trachomatis strains to advance drug and/or vaccine development in humans. 